\title{
UNA REVISIÓN DE LA LITERATURA ACADÉMICA SOBRE LA INDEPENDENCIA PARTIDARIA: EXPLICACIONES, HALLAZGOS Y DESAFÍOS ${ }^{1}$
}

\author{
Gerardo Isaac Cisneros Yescas ${ }^{2}$
}

\section{Introducción}

La identificación partidista es un concepto que ha generado importantes debates en el campo del comportamiento electoral desde que surgió en la década de 1960. El concepto centró su atención en la importancia del vínculo que tiene un votante con un partido político para entender su comportamiento en los procesos electorales (Campbell et al, 1965). En años más recientes, distintas investigaciones sumaron al debate la declinación del lazo partidario en varias partes del mundo (Hagopian, 1998; Wattenberg, 1999; Dalton, McAllister y Wattenberg, 2000; Dalton, 1984, 2012 y 2013), enfatizando la importancia de considerar no solo a los electores identificados con un partido político, sino también a aquellos que se declaran independientes o no simpatizantes de los partidos políticos.

Investigaciones más actuales señalan que la identificación partidista se ha debilitado ampliamente no solo en Estados Unidos sino también en distintos países europeos como Gran Bretaña (Clarke, 1998), Alemania (Dalton, 2012),

\footnotetext{
${ }^{1}$ Este documento es producto de la tesis para obtener el grado de doctor en Investigación de Ciencia Política por la Facultad Latinoamericana de Ciencias Sociales y contó con el apoyo del Consejo Nacional de Ciencia y Tecnología de México.

${ }^{2}$ Actualmente es asesor en el Instituto Electoral del Distrito Federal, licenciado en Ciencias de la Comunicación por la Universidad Nacional Autónoma de México, maestro en Ciencias Sociales y doctor en Investigación en Ciencia Política por la Facultad Latinoamericana de Ciencias Sociales.
} 
Suecia (Holmberg, 2007), Italia, Austria, Bélgica, Dinamarca, Finlandia, Holanda, Luxemburgo, Irlanda, Islandia, Francia, Austria, Suiza y Japón (Dalton, 1984, 2000 y 2013), así como en países de América Latina como Costa Rica (Sánchez, 2002), Uruguay, Colombia (Giacometti, 2006), Brasil, MéxiCo, Argentina, Chile (Brussino y Vaggione, 1995; Hagopian, 1998; Temkin et al, 2008; Estrada, 2006) y Nicaragua (Saldomando y Cuadra, 2011).

En consecuencia, se han generado diversas aproximaciones teóricas acerca del estudio de los electores que no se identifican con un partido político. Este artículo expone los distintos ejes de la discusión en torno a la independencia partidista, abordando el debate en el orden que a continuación se menciona: i) causas y consecuencias del declinamiento de la identificación partidista; ii) la caracterización del elector independiente desde la postura clásica de la identificación partidista; iii) la caracterización a partir de la revisión del planteamiento clásico que distingue a los independientes puros de los inclinados y; iv) la postura de la movilidad cognitiva como alternativa a lo planteado por la postura clásica.

\section{La discusión sobre la declinación partidista}

En la literatura académica existe un debate en torno a la existencia del desalineamiento partidista o no, es decir, hay argumentos a favor y en contra de la presencia de este fenómeno. La perspectiva que abona en favor de su existencia señala a grandes rasgos tres causas principales: a) desempeño de los partidos políticos, b) cambio generacional y, c) modernización.

Respecto al desempeño partidista, se enfatiza la pérdida de fuerza de los partidos para integrar a los ciudadanos en las decisiones de gobierno y en las campañas, generando actitudes neutrales o de hostilidad hacia ellos (Wattenberg, 1999: 4-5; Nie, Verba y Petrocik, 1979). También se ha destacado que factores de corto plazo inducen a los votantes a 
cambiar de preferencia política de manera continua (Fiorina, 1981; Hagopian, 1998) y, en ocasiones, se ha hecho responsable a las élites políticas de este decrecimiento en la identificación debido a la polarización que generan (Miller y Wattenberg, 1983), así como al deficiente trabajo -percibido por los ciudadanos- de parte de los partidos (Clarke, Dutt y Kornberg, 1993).

Otra explicación de este fenómeno es el cambio generacional. Se argumenta que la caída de la identificación está vinculada al aumento de ciudadanos jóvenes no cercanos a ningún partido político, lo cual, posteriormente, se traduce en un distanciamiento en la edad adulta (Inglehart, 1970; Nie, Verba y Petrocik, 1979).

Finalmente, una de las explicaciones que ha cobrado mayor relevancia y que muestra evidencia empírica del cambio es la de la movilización cognitiva. Esta perspectiva señala que el proceso de modernización en las democracias avanzadas ha erosionado los lazos hacia los grupos sociales -producto de la expansión de la educación y de la reducción en los costos para adquirir información a través de los medios de comunicación-, afectando a los partidos políticos debido a que los ciudadanos tienen un mayor nivel de sofisticación y no necesitan de los lazos partidistas para tomar sus decisiones políticas (Dalton, 1984).

En contraste con los argumentos anteriores, se ha puesto en duda el fenómeno del desalineamiento partidista, afirmando que, al menos para Estados Unidos, la identificación con algún partido político no ha perdido relevancia ni tampoco ha decrecido en el tiempo (Miller, 1991). Se señala que si los independientes son tratados como un grupo heterogéneo compuesto por independientes inclinados e independientes puros (la etiqueta de "independientes inclinados" se refiere a que estos individuos declaran que no se identificaban con un partido, pero tienen inclinación hacia alguno de ellos. Por su parte, los independientes puros son aquellos que no se identifican con algún partido y tampoco se inclinan hacia alguno de ellos) y si, además, se considera 
a los primeros como partidistas, la declinación virtualmente desaparece (Petrocik, 2008). Esta afirmación se sostiene en la premisa de que los "verdaderos" independientes son un segmento muy pequeño del electorado y se descarta que aquellos que se autodefinen como no identificados y declaran posteriormente cercanía con algún partido político deban ser tratados en las investigaciones como independientes, dado que sí sostienen una identificación con un partido, a pesar de no manifestarla explícitamente. Estos trabajos coinciden en el cuestionamiento al fenómeno del declinamiento partidista y afirman que el crecimiento de los electores no identificados con un partido político es en realidad un mito (Wolfinger et al, 1977; Keith et al, 1992; Magleby et al, 2011).

Un aspecto también debatido es la importancia y las implicaciones que tiene la alta presencia de independientes en el sistema político. Algunos autores han señalado que el aumento de los electores independientes puede conducir al colapso y final del sistema de partidos o a la pérdida de la democracia por la inestabilidad política (Burnham, 1970; Pomper, 1977, citado en Magleby et al. 2011). En un tono menos catastrófico, también se argumenta que, cuando se erosiona el lazo hacia un partido político, se dispara la volatilidad electoral (Dennis, 1988; Hagopian 2008; Alcántara, 2004) y se fortalece la idea de la generación de un partido nuevo (Abramson et al. 1983). Es así que el sufragio de estos electores se vuelve una pieza clave para los partidos políticos (Nie, Verba y Petrocik, 1979; Abramowitz, 2009), los cuales pueden ocasionar cambios repentinos en el apoyo partidista de elección a elección (Dalton, McAllister y Wattenberg, 2000; Dalton, 2007; Abramson et al., 1983). Asimismo, se incrementa el voto dividido -consiste en sufragar por diferentes partidos en los distintos niveles de gobierno en la misma elección (Dalton, McAllister y Wattenberg, 2000; Nie, Verba y Petrocik, 1979)-, se retrasa la decisión de voto a unos días antes de la elección (Dassonneville, 2012; Dalton, 1984, 2000 y 2013; Cisneros, 2016) e 
inclusive se incrementa la posibilidad de abstención el día de las elecciones (Hagopian, 1998; Giacometti, 2006; Sánchez, 2002).

Del panorama expuesto es posible extraer una visión a veces pesimista y poco alentadora de los votantes independientes, pues pueden ser susceptibles a propuestas carismáticas o en ocasiones no estar lo suficientemente informados para tomar decisiones políticas. Aunque también es posible tener una visión positiva de ellos, dada su proclividad a realizar un voto no ligado al sentimiento afectivo hacia un partido político. Las diferentes perspectivas que se tienen sobre las consecuencias que podrian generar los no identificados solo pueden entenderse a través del perfil que se ha conformado en relación a quiénes son estos electores y qué características los distinguen. El retrato de estos electores permitirá observar que no son "peligrosos" como en ocasiones se sugiere.

\section{La visión clásica de la independencia partidista}

Los estudios acerca de los electores sin identificación partidista comenzaron a mediados del siglo pasado y se enfocaron fundamentalmente en el electorado estadounidense. En un inicio se señalaba que los independientes eran electores educados y en ocasiones con mayor nivel de escolaridad que los partidistas, pero con una limitada participación electoral (Hasting, 1953; Eldersveld, 1952). George Gallup en 1948 reportaba que "entre más nivel alcanzara el votante en la escala de educación existía mayor probabilidad de que fuera independiente" (Gallup, 1948, citado en Keith et al., 1992).

Esta primera aproximación fue seguida por otros investigadores que destacaron otras características señalando que los votantes con alto interés en la política y con bajo nivel de partidismo, es decir, independientes involucrados políticamente, eran un caso desviante (Lazarsfeld, et al., 1954 citado en Dennis, 1988; 91). Es a partir de la publica- 
ción del libro The American Voter -trabajo clásico que dio origen al concepto de identificación partidista- cuando se exponen de manera clara y sistemática los atributos fundamentales de los electores no identificados con un partido político.

En dicha investigación se señala que, más allá del ideal normativo del ciudadano independiente, atento a la política y preocupado por el trabajo del gobierno, los no identificados con un partido político tienden a estar "mucho menos involucrados en la política. Poseen un pobre conocimiento sobre los temas políticos, una imagen débil acerca de los candidatos en contienda, un interés menor en las campañas, así como una preocupación escasa sobre los resultados. Su elección entre los candidatos contendientes se realiza casi al final de las campañas y ésta se encuentra poco relacionada con la evaluación de los elementos de la política nacional" (Campbell et al., 1965: 143).

Este retrato de los independientes deja en claro que no son un grupo con características "virtuosas", dado su bajo nivel de interés en la política, su pobre conocimiento de los asuntos públicos y la baja participación atribuida a ellos. Esta ausencia de involucramiento político encajaba plenamente con el argumento teórico expuesto por estos autores, pues ellos concibieron la identificación partidista como el principal motor que estructura "las percepciones y las actitudes respecto de la política" (Campbell et al., 1965: 97). Así, la identificación partidista fue definida por estos autores como "la orientación afectiva de un individuo hacia un grupo-objeto importante en su ambiente", siendo, "los partidos políticos [...] el grupo hacia el cual el individuo podría desarrollar una identificación, positiva o negativa, con algún grado de intensidad" (Campbell et al., 1965: 121-122). Enfatizaban que la preferencia por un partido político era muy estable de elección a elección, lo cual sugería que un número alto de votantes se mantenía cercano a un partido de manera persistente en el tiempo, siendo la independencia un fenómeno marginal. 
La definición señalada, sumada a las características expuestas por estos autores acerca de la independencia partidista, hace posible comprender que, desde esta perspectiva, la carencia de identificación con un partido político genera un distanciamiento respecto de los asuntos públicos, y esta es una de las principales condiciones para la activación política en apoyo del partido hacia el cual se dirige el sentimiento de afecto político. La manera a través de la cual se realizó la medición de la independencia e identificación partidistas fue a través de la autoclasificación de los individuos en un eje continuo de valores. La pregunta que se utilizó originalmente fue la siguiente: "hablando de manera general, iusted se considera republicano, demócrata, independiente o qué?" A aquellos que se autodenominaban independientes se les interrogaba si se "consideraban más cercanos al partido republicano o al demócrata", para identificar la inclinación del votante, aunque fuera independiente.

En suma, la visión clásica muestra que los independientes son un grupo de electores con un bajo nivel de involucramiento político y, por consiguiente, desinteresados de los asuntos públicos. La ausencia del lazo partidista es la causa principal por la que estos electores quedan al margen de la situación política, ya que no poseen el marco de referencia que brinda la identificación con un partido político.

\section{Crítica a la postura clásica:}

\section{los distintos tipos de independientes}

La pregunta latente en la mayoria de las investigaciones posteriores que abordaban el tema de la independencia partidista era si todos los independientes eran como lo establecía la postura clásica o si podían distinguirse diferencias en su interior. En caso de que sí hubiera distinciones, se cuestionaba en qué medida se alejaban de la perspectiva anterior y cuáles eran las características asociadas a ellos. 
El principal derrotero de los argumentos se concentró en distinguir entre los independientes puros y los inclinados. El argumento principal de esta perspectiva se encuentra en el libro The Myth of the Independent Voter, donde se retoma la escala clásica de siete categorías en las cuales quedaban agrupados los individuos (las categorías de identificación partidista son: i) demócratas fuertes; ii) demócratas débiles; iii) independientes demócratas; iv) independientes puros; v) independientes republicanos; vi) republicanos débiles; vii) republicanos fuertes) y distingue las actitudes y comportamientos al interior del grupo de no identificados. Keith et al. (1992: 4) señalan que "los independientes, definidos de manera inclusiva, tienen poco en común. Son más diversos que los republicanos y demócratas. La mayoría de ellos no están comprometidos con un partido y por lo tanto no son un bloque homogéneo. Son en gran parte demócratas o republicanos de clóset, respectivamente".

Los hallazgos principales muestran que, al distinguir a los independientes entre puros e inclinados, se encuentra que los independientes puros están menos interesados, informados y activos respecto de los partidos. Tienen poca propensión a expresar opiniones acerca de las principales instituciones y líderes políticos y, además, están menos comprometidos cívicamente (Keith et al., 1992: 59; Korey y Lascher, 2010: 3). En contraste, los independientes inclinados no solo están más involucrados en los temas políticos, sino que, en ocasiones, superan a los partidistas débiles en este rubro (Keith et. al., 1992: 59), pues, como señalan Lascher y Korey (2011), los inclinados, en general, se comportan como partidistas.

Por lo anterior, se argumenta que los independientes inclinados tienden a tomar posición apoyando al partido más cercano a su preferencia y lo hacen de esa manera porque no son electores neutrales, sino partidistas. Su partidismo "es evidente no solo en su voto, sino también en la estabilidad de su identificación y en sus actitudes hacia los parti- 
dos políticos" (Keith et al., 1992: 111). Es solo que son partidistas "de clóset".

Entonces, ipor qué ciertos individuos se definen como independientes si no lo son? Petrocik (2008) sugiere que la etiqueta de independientes es una forma de presentación y no una manera adecuada de referirse a ellos, pues su comportamiento y actitudes se asemejan más a las de los partidistas que a las de los independientes puros. En ese sentido, Miller (1991) señala, como posible explicación a esta anomalía, que autodefinirse como independiente resulta más aceptable socialmente que declararse partidario de algún partido político.

Por lo anterior, esta perspectiva no se distancia por completo del planteamiento clásico, sino que lo desmenuza. La idea general que subyace es que la manera más apropiada de considerar a los electores no identificados con un partido político es separándolos en dos grupos, por un lado los independientes puros y, por el otro, los inclinados. A partir de esta segmentación, se afirma desde esta visión teórica que la caracterización hecha por los académicos de la Escuela de Michigan corresponde solamente al retrato de los independientes puros y no al de los inclinados. Estos hallazgos implican que no es posible hablar de un bloque homogéneo de individuos no identificados sino que hay que distinguir diferencias al interior del grupo.

\section{La perspectiva de la movilidad cognitiva en la independencia partidista}

La teoría de la movilidad cognitiva (Dalton, 1984 y 2007) explica de otra manera la independencia partidista y considera que las características de estos electores se han modificado a lo largo del tiempo, argumentando que en las democracias avanzadas ha surgido un nuevo tipo de elector independiente con alta escolaridad y con un marcado interés por los asuntos políticos.

La teoria de movilidad cognitiva se apoya en el argumento de Shively (1979) y señala que la identificación par- 
tidista opera como un marco funcional que permite a los electores poco informados comprender los sucesos políticos. Desde esta perspectiva, la identificación con un partido político es una pantalla perceptual, a través de la cual los individuos interpretan y evalúan sus experiencias políticas. Esto debido a que las claves partidistas son un heurístico eficiente que permite a los ciudadanos decidir qué políticas y candidatos apoyar (Dalton, 2007: 275).

A pesar de la evidencia sobre el valor funcional de la identificación partidista para el ciudadano promedio, Dalton (1984: 285) señala que, aunque muchos votantes continúan considerando en su decisión las claves emanadas de los partidos políticos, la necesidad de dicho lazo ha declinado mientras que las habilidades políticas de los votantes se han incrementado y los costos para adquirir información han decrecido. Este fenómeno es producto de la expansión de la educación en las democracias industriales avanzadas que ha producido un cambio cualitativo en la sofisticación política del electorado. Al mismo tiempo, se ha experimentado una explosión de información a través de los medios de comunicación, lo que redujo los costos para adquirirla.

Así, la movilización cognitiva significa que más ciudadanos poseen los recursos y habilidades políticas necesarios para lidiar mejor con las complejidades de la política y tomar sus propias decisiones sin dependencia del lazo partidista afectivo habitual $\mathrm{u}$ otras claves externas. Por consiguiente, el desalineamiento del electorado a largo plazo en las democracias avanzadas podría vincularse con el proceso paralelo de aumento de la movilidad cognitiva. La evidencia sobre varios países de Europa, Estados Unidos y Canadá, principalmente, sugiere que la movilidad cognitiva favorece el declinamiento de las identidades partidistas y crea un nuevo grupo de independientes sofisticados, distintos de los que la visión clásica de la escuela de Michigan catalogaba como limitados políticamente.

Dado que la movilidad cognitiva no es una característica observable en los electores, se ideó una manera de me- 
dir dicha propiedad en los individuos a través de un índice (Dalton, 1984). Este está compuesto por la combinación de dos factores: la educación -que representa el componente de habilidades- y el interés en la política -que representa el componente motivacional para aplicar esas habilidades a la política-. Los altamente movilizados son aquellos que poseen ambas características y pueden lidiar con las complejidades de la política por sus propios medios, mientras que los electores con baja movilidad cognitiva no pueden.

$\mathrm{El}$ índice de movilidad cognitiva fue combinado con la identificación partidista y generó con ello otro índice denominado "cognitivo-partidista" (Dalton, 2013), del cual se desprenden cuatro perfiles electorales: partidistas rituales, partidistas cognitivos, apartidistas y apolíticos. Dentro del grupo de los votantes independientes se encuentran los dos últimos.

Los apolíticos son electores con baja movilidad cognitiva, no involucrados en cuestiones relacionadas con la política y con un interés casi nulo en esos asuntos, lo cual los emparenta con la definición clásica del votante independiente señalada por los teóricos de la escuela de Michigan. Por otro lado, los apartidistas son individuos que tampoco mantienen cercanía con ningún partido, pero sí se encuentran involucrados en los temas políticos. Debido a su nivel de movilidad cognitiva alto, tienen las habilidades necesarias para orientarse políticamente sin dependencia de las claves partidistas, por lo que pueden participar tanto en las elecciones como en otras actividades políticas (Dalton, 1984, 2007 y 2013).

Los principales hallazgos sobre estos electores señalan que los apartidistas suelen ser altamente volátiles de elección a elección, deciden por quién votar durante el periodo de campañas electorales -no como los partidistas, que lo hacen antes de iniciar el proceso-, dividen su voto con mayor frecuencia en la misma elección y son más propensos a apoyar a los partidos nuevos que entran al proceso electoral. Por otro lado, en el caso de los apolíticos, aunque 
pueden llegar a comportarse como los apartidistas, su bajo nivel de participación los coloca en los límites del proceso político, siendo electores marginales que por lo general deciden no participar en las actividades políticas, ya que carecen tanto de movilidad cognitiva como de identificación partidista (Dalton 2013; Lisi, 2010).

A diferencia de la propuesta de Magleby et al. (2011) y Keith et al. (1992), la movilidad cognitiva no asume que los independientes involucrados, es decir, los apartidistas sean partidistas de "clóset" que ocultan su verdadera identidad autodenominándose independientes. Al contrario, esta teoria señala que su alta volatilidad electoral de elección a elección es la fiel muestra de que no esconden su preferencia política. De hecho, la teoría de la movilidad cognitiva señala que los independientes inclinados pueden favorecer a un partido en unos comicios electorales y en los siguientes pueden volver a apoyar a ese mismo partido, a otro o abstenerse (Dalton, 2013).

Asimismo, se argumenta que los independientes puros son los que la teoría de la movilidad cognitiva reconoce como apolíticos, mientras que la mayoría de los independientes inclinados son apartidistas. Por esa razón, estos últimos suelen estar más interesados políticamente y más activos en las elecciones. La diferencia sustancial entre ambos tipos de no identificados es la movilidad cognitiva y no el supuesto partidismo oculto, como lo señala la perspectiva comentada previamente.

\section{Los desafíos en esta agenda de investigación}

El estudio de los electores independientes plantea una agenda de investigación necesaria dentro de la ciencia política y, en específico, dentro del estudio del comportamiento político. Las perspectivas de análisis presentadas enfatizan los rasgos que caracterizan a esos individuos, pero colocan poco énfasis en las consecuencias que ello genera en situaciones específicas. Algunos desafíos que es necesa- 
rio atender dentro de la literatura de la independencia partidista a partir de lo comentado son las siguientes.

a. Analizar el comportamiento político-electoral de estos electores, es decir, explicar en qué situaciones este tipo de electores pueden optar por las distintas opciones políticas o incluso abstenerse de votar o anular el voto.

b. Analizar las implicaciones que tiene el hecho de que los independientes, a pesar de ser electores no identificados con algún partido político, sí poseen una ideología, ya sea de izquierda, de derecha o de centro, lo cual puede conducirlos a apoyar proyectos políticos específicos según la tendencia ideológica $y$, así, favorecer o perjudicar el triunfo de alguna opción política.

c. Evaluar los efectos del contexto en el comportamiento de los independientes. Es decir, comparar si en las democracias nuevas, como las latinoamericanas, las características de los independientes son distintas respecto de democracias de más larga data, como la de los Estados Unidos de América e inclusive las de Europa.

d. Además de la participación electoral, existen otras formas de expresión política de las cuales también son partícipes los independientes, como las formas de participación no institucionalizadas. Quizá en comportamiento no electoral como acciones de protesta, movilizaciones, mítines, reuniones vecinales, boicots, etc. puedan apreciarse con mayor nitidez los efectos de la movilidad cognitiva o distinciones entre los independientes puros y los inclinados.

e. Otra tarea pendiente sobre el análisis de los independientes es la evaluación que ellos mismos tienen de su propia independencia política atendiendo lo apuntado por Dennis (1988). Es pertinente analizar si consideran la no identificación partidista de una manera positiva o, por el contrario, si en realidad están ocultando su preferencia política. De entrada, podría plantearse como hipótesis que los independientes tienen una concepción positiva de sí mismos y sobre todo liberal de 
la política debido a que la independencia partidista en sí misma apela a la libertad individual de elegir sin estar ligado a un agente político específico externo.

f. Finalmente, existen investigaciones que señalan que la evaluación del desempeño del gobierno es un criterio de decisión fundamental en la decisión electoral de los independientes (Cisneros, 2015; Dalton, 2013). Ante ello, un cuestionamiento que surge es ¿qué hay en esa evaluación del desempeño? ¿cuál es el contenido que los electores depositan en esa evaluación? Quizá ahí radiquen diferencias sustantivas entre los diferentes tipos que se han mostrado en la literatura.

Las investigaciones que abordan el tema de la independencia partidista podrían seguir algunos de los ejes señalados. Sin embargo, debe recalcarse, tal y como lo establece la literatura sobre el tema, la pertinencia de segmentar al electorado en independientes y partidistas cuando se analice su comportamiento y considerar algún criterio de división al interior de los no identificados, ya sea a través de la división de inclinados y puros o de apartidistas y apolíticos, eso no sin antes contemplar que el uso de cualquiera de las dos aproximaciones teóricas tiene implicaciones analíticas y empíricas muy diferentes.

\section{Referencias}

Abramowitz, Alan 2009 "The Myth of Independent Voter Revisited." Center for Politics, August 20.

Abramson, Paul; John Aldrich y David Rohde 1983 Change and Continuity in the 1980 Elections. Washington: CQ Press.

Alcántara, Manuel 2004 Partidos Políticos en América Latina: precisiones conceptuales, estado actual y retos futuros. América Latina. Barcelona: Cidob.

Brussino, Silvina y Juan Marco Vaggione 1995 "El apartidismo y el politismo: un análisis a partir de la sensación de falta de poder." Anuario, n 3, sección 3: Sociología, política e historia. 
Burnham, Walter 1970 Critical Elections and the Mainsprings of American Politics. New York: W. W. Norton.

Campbell, Angus; Philip Converse; Warren Miller y Donald Stokes 1965 The American Voter. New York: John Wiley and Sons.

Cisneros, Isaac 2015 La decisión electoral del votante independiente en América Latina: movilidad cognitiva o independencia partidista. Tesis de Doctorado en Investigación en Ciencia Política. México: Flacso.

2016 "Votante anulista: independencia partidista y alta movilidad cognitiva. El caso de la elección federal de 2009 en México." Colombia Internacional, $\mathrm{n}^{\circ} 86$ (Abril): 51-80.

Clarke, Harold y Marianne Stewart 1998 "The Decline of Parties in the Minds of Citizen." Annual Review of Political Science, 357-78.

Dalton, Russell 1984 "Cognitive Mobilization and Partisan Dealignment in Advanced Industrial Democracies." The Journal of Politics 46 (1): 264-84.

2000 "The Decline of Party Identifications." En Parties without Partisans. Political Change in Advanced Industrial Democracies, 19-36. New York: Oxford University Press.

2007 "Partisan Mobilization, Cognitive Mobilization and the Changing American Electorate." Electoral Studies, $\mathrm{n}^{0} 26$ : 247-86.

2012 "Apartisans and the Changing German Electorate." Electoral Studies, $\mathrm{n}^{\circ}$ 31: 35-45.

2013a "The Apartisan American". En Russell Dalton, The Apartisan American: Dealignment and Changing Electoral Politics. Oaks: CQ Press/ Los Angeles: SAGE.

2013b The Apartisan American: Dealignment and Changing Electoral Politics. Oaks: CQ Press/ Los Angeles: SAGE.

Dalton, Russell; Ian McAllister y Martin Wattenberg 2000 "The Consequences of Partisan Dealignment." En Parties without Partisans. Political Change in Advanced Industrial Democracies, 37-63. New York: Oxford University Press.

Dassonneville, Ruth 2012 "Cognitive Mobilization and Vote Intention Switching. An Event History Analysis of Electoral Volatility 
in the 2009 German Election Campaign." Paper preparado para la presentación de la 108 reunión anual de la Asociación Americana de Ciencia Política.

Dennis, Jack 1988a "Political Independence in America, Part I: On Being an Independent Partisan Supporter." British Journal of Political Science 18 (1): 77-109.

1988b "Political Independence in America, Part II: Towards a Theory." British Journal of Political Science 18 (2): 197-219.

Eldersveld, Samuel 1952 "The Independent Vote: Measurement, Characteristics, and Implications for Party Strategy." The American Political Science Review 46 (3): 732-53.

Estrada, Luis 2006 "Determinantes y características de los independientes en México." Política y Gobierno XIII (1): 149-73.

Fiorina, Morris 1981 Retrospective Voting in American National Elections. London: New Haven.

Giacometti, Enrico 2006 "Desalineación electoral y nuevos escenarios políticos. Un estudio comparado sobre los casos de Colombia, Costa Rica y Uruguay." Encuentro de Latinoamericanistas Españoles (diciembre 2006 - Santander). Viejas y nuevas alianzas entre América Latina y España, 2006, España. CEEIB, pp. 242-251..

Hagopian, Frances 1998 "Democracy and Political Representation in Latin America in the 1990s: Pause, Reorganization, or Decline?" En Fault Lines of Democracy in Post-Transition Latin America, 99-143. Miami: North-South Center Press.

Hasting, Philip 1953 "The Independent Voter in 1952: A Study of Pittsfield, Massachusetts." The American Political Science Review 47 (3): 805-10.

Holmberg, Sören 2007 "Partisanship Reconsidered." En The Oxford Handbook of Political Behaviour, 557-60. Oxford: Oxford University Press.

Inglehart, Ronald 1970 "Cognitive Mobilization and European Identity" 3 (1): 45-70.

Keith, Bruce; David Magleby; Candice Nelson; Mark Westlye; Elizabeth Orr y Raymond Wolfinger 1992 The Myth of the Independent Voter. Berkeley: University of California Press. 
Korey, John y Edward Lascher 2010 "Political Independents and Civic Engagement in California." Paper presentado en la Conferencia Anual de la Western Political Science Association, Abril.

Lascher, Edward y John Korey 2011 "The Myth of the Independent Voter, California Style." The California Journal of Politics and Policy 3 (1).

Lisi, Marco 2010 "The Consequences of Cognitive Mobilization in Comparative Perspective: Political Sophistication and Voting Behavior in Old and New Democracies." Paper prepared for presentation at the Annual Meeting of the American Political Science Association, September 2-5, 2010, Washington, D.C.

Magleby, David; Candice Nelson y Mark Westlye 2011 "The Myth of the Independent Voter Revisited." En Facing the Challenge of Democracy: Explorations in the Analysis of Public Opinion and Political Participation, 238-63. Princeton: Princeton University Press.

Miller, Arthur y Martin Wattenberg 1983 "Measuring Party Identification: Independent or No Partisan Preference?" American Journal of Political Science 27 (1): 106-21.

Miller, Warren 1991 "Party Identification, Realignment and Party Voting: Back to the Basics." American Political Science Review 85 (June): 557-68.

Nie, Norman; Sidney Verba y John Petrocik 1979 "The Decline of Partisanship." En The Changing American Voter, 47-73. Cambridge: Harvard University Press.

Petrocik, John 1989 "An Expected Party Vote: New Data for an Old Concept." American Journal of Political Science 33 (February). Paper prepared for the Shambaugh Conference on "The American Voter: Change or Continuity over the Last Fifty Years?". Department of Political Science, Universidad de Iowa. May 8-10, 2008.

Saldomando, Ángel; Sofía Montenegro; Carlos Chamorro y Elvira Cuadra 2011 "Los votantes independientes en Nicaragua."

Sánchez, Fernando 2002 "Desalineamiento electoral en Costa Rica." Ciencias Sociales IV (98): 29-52. 
Shively, Phillips 1979 "The Development of Party Identification among Adults: Exploration of a Functional Model." The American Political Science Review 73 (4): 1039-54.

Temkin, Benjamín, Sandra Solano y José del Tronco 2008 "Explorando el 'apartidismo' en México: ¿apartidistas o apolíticos?" América Latina Hoy 50 (diciembre): 119-45.

Wattenberg, Martin 1999 The Decline of American Political Parties, 1952-1996. Cambridge: Harvard University Press. 